\title{
Outer-membrane antigen expression by Moraxella (Branhamella) catarrhalis influences pulmonary clearance
}

\author{
JENNELLE M. KYD, ALLAN W. CRIPPS and TIMOTHY F. MURPHY \\ Faculty of Applied Science, University of Canberra, Belconnen, Australian Capital Territory 2616, Australia and \\ * Division of infectious Diseases of the Department of Medicine and Department of Microbiology, State \\ University of New York at Buffalo, Buffalo, New York, USA
}

\begin{abstract}
Moraxella (Branhamella) catarrhalis is a common respiratory tract pathogen in man. The bacterium shows a strong tendency to form aggregates in vitro. A variant strain of $M$. catarrhalis that showed a reduced tendency to form aggregates was selected by successive in-vitro passage in broth culture from which aggregates had settled. The nonclumping variant strain showed alteration in expression of outer-membrane antigens, including the HMW-OMP, an outer-membrane protein of $c .200 \mathrm{kDa}$, outer-membrane protein CD and lipo-oligosaccharide. A mouse model for pulmonary challenge with $M$. catarrhalis revealed significant differences in the rate of clearance of the isogenic variant strains from the lung. The parent strain caused enhanced recruitment of neutrophils to the lung and more rapid clearance of bacteria from the lungs in comparison to the nonclumping variant. It is concluded that alteration of expression of surface molecules by $M$. catarrhalis has a significant impact in an in-vivo model of pulmonary clearance.
\end{abstract}

\section{Introduction}

Moraxella (Branhamella) catarrhalis is a common cause of respiratory tract infection in man. The bacterium causes c. $15 \%$ of episodes of otitis media, an estimate based on culture of middle ear fluid obtained by tympanocentesis [1]. M. catarrhalis also causes lower respiratory tract infections in adults with chronic obstructive pulmonary disease (COPD) [2]. In one study, c. $30 \%$ of purulent exacerbations of COPD were caused by $M$. catarrhalis [3]. The recognition of $M$. catarrhalis as a human respiratory tract pathogen has stimulated interest in surface antigens and their role in the pathogenesis of infection.

M. catarrhalis shows a strong tendency to form aggregates when growing in broth culture and when suspended in buffers. To facilitate studies in vitro, a variant strain that demonstrated a reduced tendency to form aggregates was selected by passage in vitro in broth culture. Analysis of the parent and variant strains has revealed that the non-clumping variant demonstrates alterations in expression of outer-membrane antigens.

Received 3 April 1997; revised version accepted 4 July 1997.

Corresponding author: Professor T. F. Murphy.

e-mail: murphyt@acsu.buffalo.edu
Clearance of $M$. catarrhalis from the lungs of mice has been used to study the pathogenesis of infection and to evaluate potential vaccine antigens [4-9]. The aims of the present study were to characterise two isogenic non-clumping and parent strains of $M$. catarrhalis and to study the behaviour of these strains in the mouse pulmonary clearance model.

\section{Materials and methods}

\section{Bacterial strains and growth conditions}

M. catarrhalis strain 4223 (kindly provided by $H$. Faden) was isolated from the middle ear fluid obtained by tympanocentesis from a child with otitis media in Buffalo, New York, USA. Strain $4223 \mathrm{NC}$ is a nonclumping variant of strain 4223. To generate strain $4223 \mathrm{NC}$, an overnight broth culture of strain 4223 was allowed to stand undisturbed at room temperature for 30 min. Bacteria settled to the bottom of the flask. A 1$\mathrm{ml}$ sample of broth from just below the surface was taken and used to inoculate a fresh broth culture. After overnight incubation with shaking, the flask was allowed to stand undisturbed and the same process was performed again. Seven successive such subcultures were performed to generate strain 4223 NC. $M$. catarrhalis strain K114 (kindly provided by Barbara Chang) was isolated from the sputum of an adult in Perth, Western Australia. 
Bacteria were grown on Brain Heart Infusion Agar supplemented with defibrinated horse blood $50 \mathrm{ml} / \mathrm{L}$ (Amadeus International, Brooklyn, Victoria, Australia) or on chocolate agar. Plates were incubated overnight at $37^{\circ} \mathrm{C}$ in air with $\mathrm{CO}_{2} 5 \%$. When bacteria were grown in broth culture, brain heart infusion broth was used.

\section{Whole bacterial cell preparations}

Bacteria from agar plates incubated overnight were suspended in PBS so that a 1 in 10 dilution yielded an optical density of $c$. 0.3 at $405 \mathrm{~nm}$. A $10-\mu 1$ volume of the undiluted suspension of bacteria was mixed with $10 \mu 1$ of sample buffer (10 mM Tris, $1 \mathrm{mM}$ EDTA, SDS $2.5 \% \mathrm{w} / \mathrm{v}$, glycerol $10 \% \mathrm{v} / \mathrm{v}, 2$-mercaptoethanol $5 \%$ $\mathrm{v} / \mathrm{v}$ ) and heated in a boiling water bath for $5 \mathrm{~min}$. The entire sample was loaded on to an SDS-PAGE gel.

\section{Isolation of outer membrane}

The outer membranes of strains 4223 and $4223 \mathrm{NC}$ were isolated by the zwittergent extraction method as described previously [10].

\section{Isolation of lipo-oligosaccharide}

The lipo-oligosaccharides (LOS) of strains 4223 and $4223 \mathrm{NC}$ were purified by a microphenol extraction method as described by Inzana [11].

\section{$S D S-P A G E$}

Samples of whole-cell lysates and zwittergent-extracted outer membrane were subjected to SDS-PAGE on 10 $20 \%$ gradient gels (BioRad) in the mini Protean II electrophoresis apparatus (BioRad) at a constant $200 \mathrm{~V}$. Gels were stained with Coomassie Blue [12].

LOS was subjected to electrophoresis by tricine-SDSPAGE as described by Lesse et al. [13].

\section{Immunoblot assay}

Antigens were transferred electrophoretically from gels to nitrocellulose by methods described previously [14]. Blots were probed with monoclonal antibodies (MAbs) which recognised outer-membrane proteins (OMPs) of $M$. catarrhalis $[15,16]$. Bands were detected with goat anti-mouse IgG-IgM conjugated to peroxidase and blots were developed with horseradish peroxidase colour developer solution (BioRad).

\section{Randomly amplified polymorphic DNA (RAPD) analysis}

RAPD was performed to confirm that strains 4223 and 4223 NC were isogenic. To prepare DNA templates, bacteria were grown on agar plates overnight. Four or five colonies were suspended in $0.1 \mathrm{ml}$ of sterile distilled water in an Eppendorf tube. Cells were centrifuged at $12000 \mathrm{~g}$ for $5 \mathrm{~min}$ at $4^{\circ} \mathrm{C}$ and the supernate was discarded. The pellet was suspended in $0.1 \mathrm{ml}$ of $1 \mathrm{M} \mathrm{NaCl}$; the cells were centrifuged as above and the supernate was discarded. Cells were suspended in TE buffer (10 mM Tris, $1 \mathrm{mM}$ EDTA, $\mathrm{pH} 8.0$ ). The cell suspension was heated at $100^{\circ} \mathrm{C}$ in a heat block for $5 \mathrm{~min}$, vortex mixed briefly and then incubated for a further $10 \mathrm{~min}$ at $100^{\circ} \mathrm{C}$. The suspension was centrifuged at $12000 \mathrm{~g}$ for $10 \mathrm{~min}$ at $4^{\circ} \mathrm{C}$ to pellet the debris. The supernate was recovered and used immediately or stored at $-20^{\circ} \mathrm{C}$ for up to 1 month. This supernate was used as the DNA template in the polymerase chain reaction (PCR).

PCR was performed in a total reaction volume of $50 \mu \mathrm{l}$. A $5-\mu \mathrm{l}$ volume of template was used in each reaction. A single primer, BR4, was used. This primer corresponds to a sequence in the gene which encodes OMP B2 (cop B) of $M$. catarrhalis strain 25240. Its sequence is: ATATTAGCCCCTGTGGCT. In addition to the template, the $\mathrm{PCR}$ reaction mixture contained $2.5 \mathrm{mM} \mathrm{MgCl}_{2}, 12.5 \mathrm{mM}$ dNTPs, $100 \mathrm{pM} \mathrm{BR} 4$ primer, PCR buffer II (Perkin Elmer; final concentration $1 \times$ ) and 1.5 units of Taq I polymerase.

The reaction mixture was heated at $94^{\circ} \mathrm{C}$ for $5 \mathrm{~min}$. The reaction was then run for 48 cycles as follows: $94^{\circ} \mathrm{C}$ for $1 \mathrm{~min}, 25^{\circ} \mathrm{C}$ for $1 \mathrm{~min}$ and $74^{\circ} \mathrm{C}$ for $4 \mathrm{~min}$. A further two cycles were carried out as follows: $94^{\circ} \mathrm{C}$ for $1 \mathrm{~min}, 25^{\circ} \mathrm{C}$ for $1 \mathrm{~min}$ and $74^{\circ} \mathrm{C}$ for $9.5 \mathrm{~min}$. This was followed by one additional incubation at $74^{\circ} \mathrm{C}$ for $30 \mathrm{~s}$. A $10-\mu \mathrm{l}$ volume of the resulting reaction mixture was added to $2 \mu 1$ of $6 \times$ sample buffer (bromophenol blue $0.25 \%$, Ficoll type $40015 \%$, Pharmacia). The samples were subjected to agarose gel electrophoresis with NuSieve (FMC BioProducts, Rockland, ME, USA) $2.1 \%$ in TAE buffer $(40 \mathrm{mM}$ tris acetate, $2 \mathrm{mM}$ $\mathrm{Na}_{2}$ EDTA). The gel was stained with ethidium bromide and bands were visualised by ultraviolet light.

\section{Electron microscopy}

Bacterial strains were examined for the presence of pili. Colonies from an agar plate that contained overnight growth were suspended in PBS. One drop of the suspension was applied to a copper grid, washed with distilled water and then stained with uranyl acetate $1 \%$ for $30 \mathrm{~s}$. Samples were examined with a Phillips electron microscope.

\section{Flow cytometry}

Flow cytometry was performed with MAbs known to recognise epitopes which are present on the surface of the intact bacterial cell. Bacteria were grown to midlogarithmic phase in broth. An equal volume of PBS containing DNAase $25 \mu \mathrm{g} / \mathrm{ml}$ (PBS-DNAase) to reduce clumping was added and the cells were harvested by centrifugation. Cells were then added to MAb diluted 
in PBS-DNAase. After incubation at $37^{\circ} \mathrm{C}$ for $1 \mathrm{~h}$, the cells were centrifuged and suspended in $200 \mu \mathrm{l}$ of antimouse IgG-IgM conjugated to fluorescein (Kirkegaard and Perry Laboratories Inc.) diluted in PBS-DNAase and incubated for $30 \mathrm{~min}$ at $37^{\circ} \mathrm{C}$. A $1.8-\mathrm{ml}$ volume of PBS-DNAase was added and the cells were subjected to flow cytometry with a FACScan (Becton-Dickinson). A total of 20000 cells was counted in a gated region corresponding to unclumped cells. Data were acquired by using an instrument status with a logarithmic mode for forward scatter, side scatter and fluorescence. A negative control was performed with each experiment and included incubation of cells with an equivalent volume of SP2 tissue culture supernate or purified ascites fluid prepared in exactly the same way as the antibodies.

\section{Monoclonal antibodies}

Various MAbs specific for epitopes on $M$. catarrhalis OMPs or LOS were used in flow cytometry or immunoblots. The specificity and sources of these are shown in Table 1.

\section{Bacterial challenge of $B A L B / c$ mice}

Specific pathogen-free (SPF) male BALB/c mice aged 6-8 weeks were used. They were maintained under SPF conditions until the start of the experiment. Bacteria were grown on agar plates overnight, harvested into PBS and washed three times in PBS. The concentration of the inoculum was estimated by measuring the optical density at $405 \mathrm{~nm}$ and was confirmed by counting the number of cfu after overnight culture of serial 10 -fold dilutions of the inoculum in duplicate.

Animals were sedated with Saffan (Pitman-Moore, Nth Ryde, NSW, Australia) $20 \mathrm{mg} / \mathrm{kg}$ body weight administered intravenously into the tail vein. A bolus inoculum of $10^{6} \mathrm{cfu}$ in PBS in a volume of $20 \mu \mathrm{l}$ was introduced into the lungs via an intratracheal cannula. Bacteria were dispersed with two $0.3-\mathrm{ml}$ volumes of air.

Animals were killed by an overdose of pentobarbital sodium administered by intraperitoneal injection. Bronchoalveolar lavage (BAL) was obtained by instilling $0.5 \mathrm{ml}$ of PBS into the lungs via a cannula placed into the trachea through a neck incision. The resulting BAL was recovered by aspiration of fluid from the intratracheal cannula. The BAL was assessed for bacterial clearance by plating of serial 10-fold dilutions for cfu determination. After obtaining BAL, the intact lungs were removed, placed in $2 \mathrm{ml}$ of PBS, and homogenised in a tissue homogeniser. The lung homogenate was assessed for the presence of bacteria by plating serial 10 -fold dilutions for cfu determination. All plating of serial dilutions was performed in duplicate.

\section{BAL fluid while cell counts}

To determine the total white cell count, BAL was centrifuged at $1500 \mathrm{~g}$ for $10 \mathrm{~min}$. The cell pellet was resuspended in $100 \mu \mathrm{l}$ of PBS and mixed with $100 \mu \mathrm{l}$ of methylene blue in acetic acid $1.5 \%(\mathrm{v} / \mathrm{v})$. Cells were counted with a haemocytometer and the cell count was calculated.

Cytospin slides were prepared to determine the percentages of neutrophils and macrophages present in BAL fluid. A $100-\mu 1$ volume of BAL fluid was mixed with $100 \mu \mathrm{l}$ of PBS and centrifuged for $10 \mathrm{~min}$ at $4.5 \mathrm{~g}$ on to a microscope slide in a cytocentrifuge (Shandon Inc., Pittsburgh, PA, USA). The slides were fixed and stained in Diff Quick (O'Malleys Vet Supplier, Cootamundra, NSW, Australia) and percentages were determined from three differential cell counts of 100 cells for each sample. Mean percentages and standard errors were calculated from the collected group data.

\section{Statistical analysis}

The data were assessed for statistical significance by an analysis of variance. The pulmonary clearance data, total numbers of white blood cells and differential cell counts were compared for statistical significance between groups and over time by a fully factorial analysis of variance (Macintosh Systat). Comparison of significance of responses over time for strain 4223 or $4223 \mathrm{NC}$ were performed by a one-way analysis of variance (Macintosh Systat).

Table 1. MAbs to outer-membrane antigens of $M$. catarrhalis

\begin{tabular}{llll}
\hline MAb & Antigenic specificity & Isotype/subclass & Reference \\
\hline 2.9F & OMP B2 (CopB) & IgG1 & 18 \\
9G10d & OMP E & IgG1 & 19 \\
7D6 & OMP CD & IgG1 & 16 \\
5E8 & OMP CD & IgM & 16 \\
1D3 & OMP CD central region & IgG1 & Unpublished observations \\
3.9H & OMP CD amino terminal region & IgG1 & Unpublished observations \\
9E9 & HMW-OMP (UspA) & IgM & 15 \\
4G5 & Lipo-oligosaccharide & IgG2a & Unpublished observations \\
\hline
\end{tabular}




\section{Results}

Characterisation of $M$. catarrhalis strains 4223 and $4223 N C$

A characteristic feature of $M$. catarrhalis is its tendency to aggregate. Successive in-vitro passage in broth culture, from which aggregates were allowed to settle by gravity, generated a non-clumping strain. This non-clumping variant, $4223 \mathrm{NC}$, demonstrated a different appearance when grown on agar plates in comparison with the parent strain, 4223. Strain $4223 \mathrm{NC}$ had a somewhat moist appearance whereas strain 4223 appeared dry. When colonies were harvested from agar plates, the parent strain, 4223, was 'sticky' and adhered readily to the glass slide used for harvesting. By contrast, strain $4223 \mathrm{NC}$ was less sticky and tended to run off the glass slide during harvesting. The nonclumping variant was more readily suspended in PBS in comparison with the parent strain which required more mixing to form a uniform suspension. These characteristics were stable in the two strains and were unchanged with in-vitro passage on agar plates.

To establish that the two variant strains were isogenic, RAPD and pulsed-field gel electrophoresis (PFGE) of DNA restricted with Spe I were performed [17]. RAPD is a PCR-based method which has been compared with PFGE and has proven to be a convenient and reliable method for typing strains of $M$. catarrhalis (Klingman et al., unpublished observations). Fig. 1 is an ethidium bromide-stained agarose gel which shows that strains 4223 and $4223 \mathrm{NC}$ yielded identical banding patterns by RAPD. In addition, DNA from the two strains was purified in agarose plugs, digested with restriction endonucleases and subjected to PFGE as described previously [17]. Both strains yielded identical banding patterns (data not shown). These results confirm that the two strains used in this study were indeed isogenic.

Whole-cell lysates of strains 4223 and $4223 \mathrm{NC}$ were prepared simultaneously by identical methods and were subjected to SDS-PAGE. Fig. 2 shows that strain 4223 contained two bands near the top of the gel (lane a) which were absent in the corresponding preparations from strain 4223NC (lane b). No other obvious differences between the two strains in banding patterns in the whole cell lysates were apparent. An immunoblot assay of whole-cell preparations with MAb 9E9 which recognises an epitope on the HMW-OMP [15] demonstrated that the band near the top of the gel was the HMW-OMP (Fig. 2, right panel).

The outer membranes of strains 4223 and $4223 \mathrm{NC}$ were purified by the zwittergent extraction method [10] and were subjected to SDS-PAGE. Fig. 2 shows that strain 4223 contained a prominent band of $c$. $200 \mathrm{kDa}$ in the outer membrane (lane c) whereas the outer membrane of strain $4223 \mathrm{NC}$ lacked this band (lane d). The HMW-OMP was absent from the

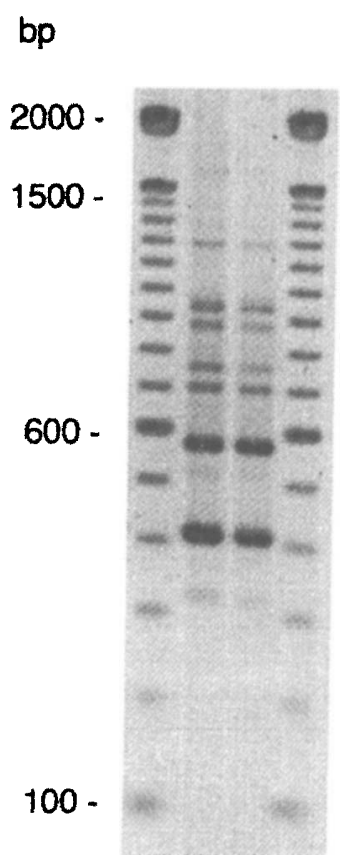

A B

Fig. 1. Ethidium bromide-stained agarose gel showing RAPD patterns of $M$. catarrhalis strains 4223 (A) and $4223 \mathrm{NC}$ (B). Mol.wt markers are in the outside lanes and are noted in $\mathrm{bp}$.

zwittergent extract of strain 4223 (lane c) in spite of the presence of the protein in the strain. This is consistent with the observation that the HMW-OMP is not recovered with the outer membrane by the zwittergent extraction method in spite of the presence of the protein in the outer membrane ([15] and authors' unpublished observation). Comparison of the zwittergent-extracted outer membranes revealed that although no bands other than the c. $200-\mathrm{kDa}$ band were absent from any one strain, differences in the intensities of several bands between the two strains were observed.

To compare the two strains with regard to the presence of other surface-exposed OMPs, flow cytometry was performed with MAbs which are known to bind to surface epitopes of OMP B2 (CopB, $c$. $82 \mathrm{kDa}$ ) [18], OMP E (c. $50 \mathrm{kDa})$ [19] and OMP CD (c. $45 \mathrm{kDa}$ ) [16]. Fig. 3 shows that MAbs 2.9F and 9G10d - which recognise epitopes on OMPs B2 and $\mathrm{E}$ respectively - yielded identical results, indicating that the epitopes on these OMPs are surface exposed to an identical degree in the two strains.

Flow cytometry with MAb 9E9 which recognises an epitope on the HMW-OMP showed markedly different results with the two strains. Strain 4223 showed a high level of expression of the epitope on its surface whereas the non-clumping variant yielded a negative result (Fig. 3). These results are consistent with those of immunoblot assay (Fig. 2), which shows an absence 


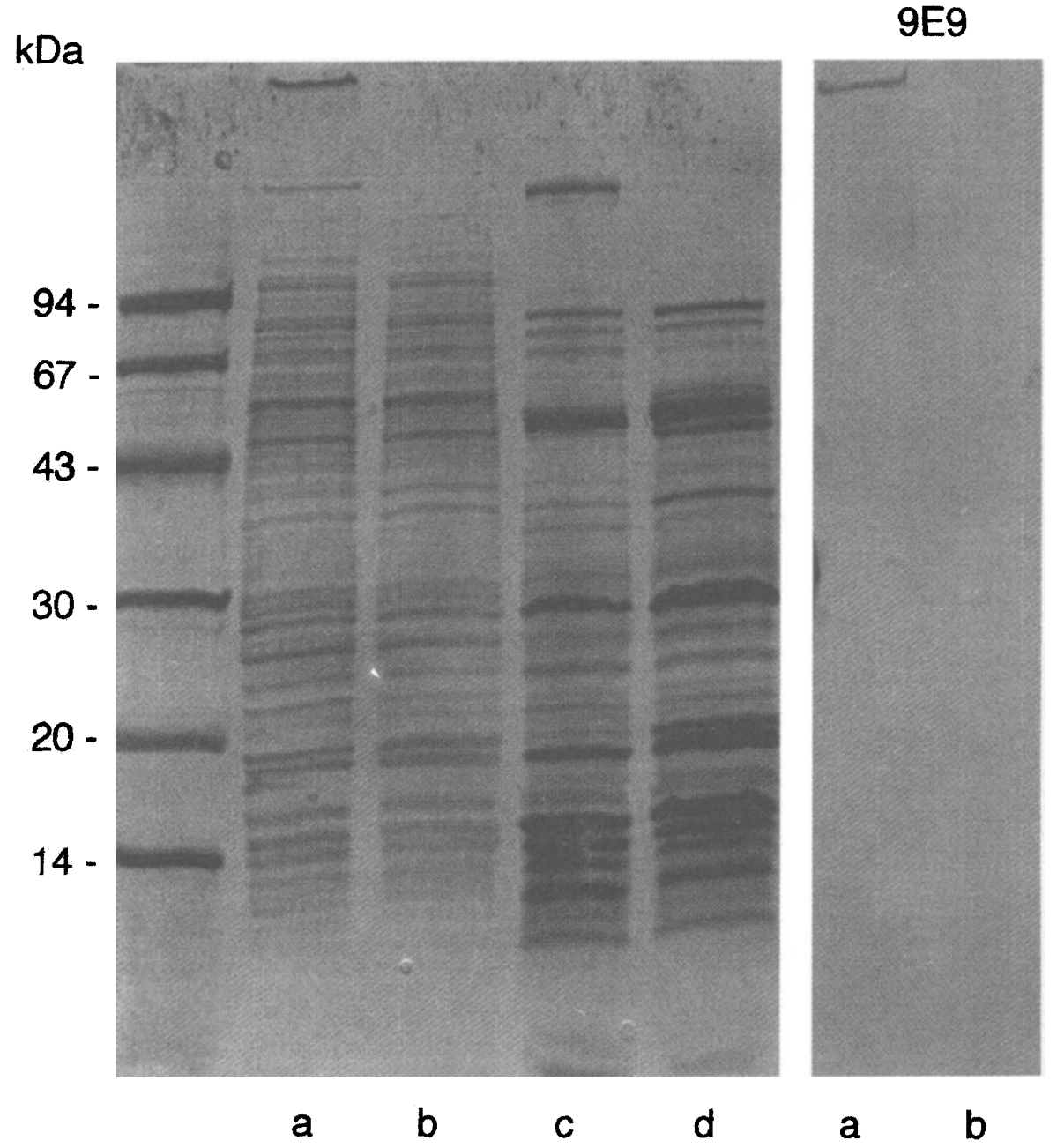

Fig. 2. Left panel: Coomassie Blue-stained 10-20\% gradient gel of whole bacterial cell lysates of $M$. catarrhalis strain 4223 (lane a) and strain 4223NC (b) and zwittergent-extracted outer membrane of strain 4223 (c) and $4223 \mathrm{NC}$ (d). Molecular mass standards are noted on the left. Right panel: immunoblot assay of whole-cell lysates of strain 4223 (a) and $4223 \mathrm{NC}$ (b) probed with MAb 9E9 which is specific for the HMW-OMP.

of the HMW-OMP detected by the antibody in strain $4223 \mathrm{NC}$.

Interestingly, two MAbs that recognise OMP CD yielded different results in flow cytometry with the two strains. Antibody 1D3, which recognises an epitope in the central region of the $\mathrm{CD}$ molecule, consistently produced a higher level of fluorescence with strain 4223 compared to the non-clumping variant, 4223NC (Fig. 3). By contrast, antibody $3.9 \mathrm{H}$, which recognises an epitope near the amino acid terminus of the $C D$ molecule, gave identical results in flow cytometry with the two strains (Fig. 3).

To determine whether the two strains expressed different amounts of OMP CD, whole-cell preparations that contained an identical number of cells based on optical density were subjected to SDS-PAGE and immunoblot assay. Four MAbs that recognise different epitopes on OMP CD were tested. Each of the four antibodies produced a band that was of equal intensity in the two strains. Fig. 4 shows the result with MAbs
5E8 and 7D6. This observation, along with the results of flow cytometry, indicated that the two strains expressed a similar amount of OMP CD but that the architecture of the outer membrane of the variant strain $4223 \mathrm{NC}$ was altered in such a way as to change the surface exposure of the central portion of the OMP CD molecule.

The LOS of strains 4223 and $4223 \mathrm{NC}$ was purified and subjected to electrophoresis on tricine gels. The LOS of both strains separated as a single band of identical mobility in tricine-SDS-PAGE. Flow cytometry was performed with a MAb that recognises an epitope on LOS of $M$. catarrhalis (kindly provided by A. Campagnari). Fig. 3 shows that the LOS epitope is more highly exposed on the bacterial surface of the parent strain than on the non-clumping variant strain.

Strains 4223 and $4223 \mathrm{NC}$ were examined by electron microscopy to determine whether they were piliate. Fig. 5 shows that the two strains did not express pili. Simultaneous examination of a third strain (K114) 

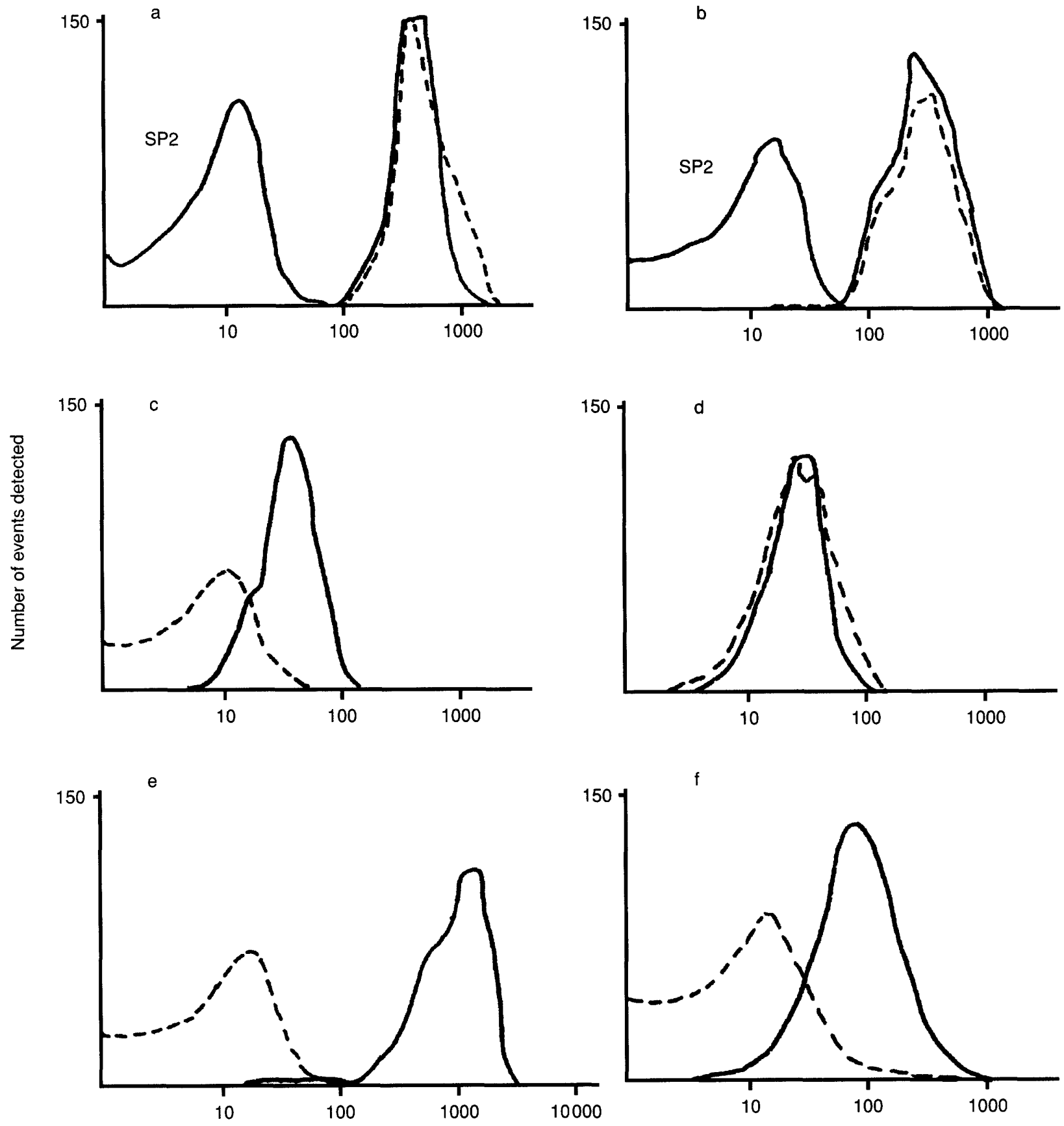

Fluorescence

Fig. 3. Results of flow cytometry with MAbs to OMPs assayed with M. catarrhalis strains 4223 and $4223 \mathrm{NC}$. Y-axes indicate events detected by the flow cytometer and $\mathrm{x}$-axes indicate fluorescence; —_, strain 4223, - - - , strain 4223 NC. The peaks marked 'SP2' represent strain 4223 assayed with SP2 tissue culture supernate as a negative control. The OMP or lipo-oligosaccharide (LOS) against which the corresponding MAb is directed as follows: a, OMP B2, antibody 2.9F; b, OMP E, antibody 9G10d; OMP CD, antibodies 1D3 (c) and 3.9H (d); e, HMW-OMP, antibody 9E9; f, LOS, antibody $4 \mathrm{G} 5$.

which is known to be piliate revealed abundant pili (Fig. 5).

\section{Pulmonary clearance}

To assess the relative rates of clearance of strains 4223 and $4223 \mathrm{NC}$ from the lung, groups of mice were challenged intratracheally with $10^{6} \mathrm{cfu}$ of either strain.
To ensure that animals were challenged with comparable inocula, the concentration of bacteria in the challenge inoculum was determined by colony counts after overnight culture of serial dilutions of the inoculum in every experiment. Optical density at $405 \mathrm{~nm}$ accurately predicted the inoculum size for both strains 4223 and $4223 \mathrm{NC}$. In addition, similar optical densities at $405 \mathrm{~nm}$ corresponded to identical 


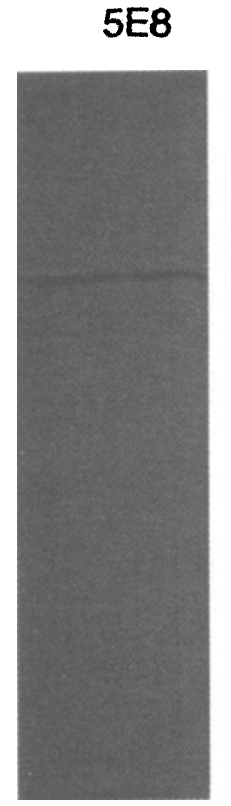

\section{$7 D 6$}

A $\quad$ B $\quad A \quad B$

Fig. 4. Immunoblot assay with whole bacterial cell lysates of $M$. catarrhalis strains 4223 (lanes A) and $4223 \mathrm{NC}$ (B). Lysates of the two strains were prepared with equivalent numbers of bacterial cells. Panels were assayed with MAbs 5E8 and 7D6 that recognise different epitopes on OMP CD.

numbers of cfu for both strains, indicating that the tendency of the bacteria to aggregate did not interfere with the ability to obtain accurate colony counts.

Bacterial clearance was measured by quantifying bacteria in BAL and lungs at 2, 4, 6 and $12 \mathrm{~h}$ after challenge. Fig. 6 shows that strain 4223 was cleared at a faster rate than $4223 \mathrm{NC}(\mathrm{p}=0.003)$. The difference was especially prominent in BAL fluid. At all time points the ratio of bacteria in BAL to bacteria in lung was higher for strain $4223 \mathrm{NC}$. These observations indicate that more bacterial cells of strain $4223 \mathrm{NC}$ remain in the airways following challenge.

\section{Recruitment of cells to the lung}

Total white blood cell counts in BAL were determined and these are shown in Fig. 7. The cell count was significantly higher in BALs of mice challenged with strain 4223 than in those challenged with $4223 \mathrm{NC}$ at all time points $(p<0.001)$. A differential cell count was determined on samples after cytospin. Fig. 7 shows that challenge with strain 4223 resulted in a significant increase $(p=0.02)$ in recruitment of neutrophils to the lungs compared to that observed with strain $4223 \mathrm{NC}$.

\section{Discussion}

A variant strain of $M$. catarrhalis that showed a reduced tendency to form aggregates was selected by successive in-vitro passage in broth culture from which aggregates had settled. When mice were challenged intratracheally with the two strains, striking differences in the rate of clearance from the lungs were observed. In an effort to interpret the significance of the altered pulmonary clearance, and variant strains were carefully characterised.

Typing of the two strains by methods based on DNA sequences confirmed that the two strains used in the study were isogenic. Analysis of the two strains with particular attention to outer-membrane antigens revealed several differences between the isogenic strains: (1) a marked reduction in expression of HMW-OMP (UspA) on the bacterial surface of the non-clumping variant; (2) absence of a $c .200-\mathrm{kDa}$ protein in the outer membrane of the non-clumping variant; (3) alteration in surface accessibility of some but not all surface epitopes on OMP CD - however, the strains

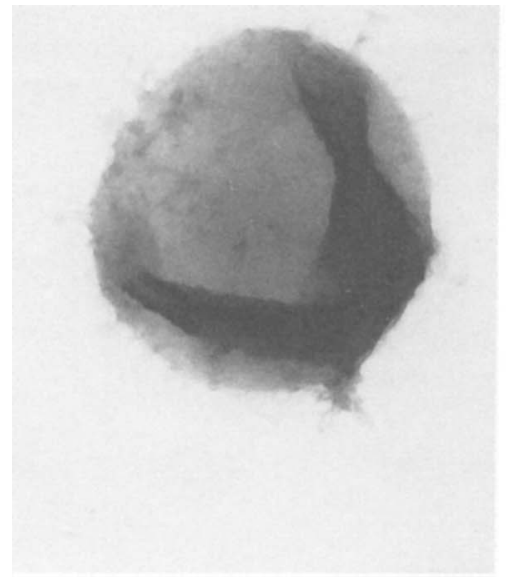

4223

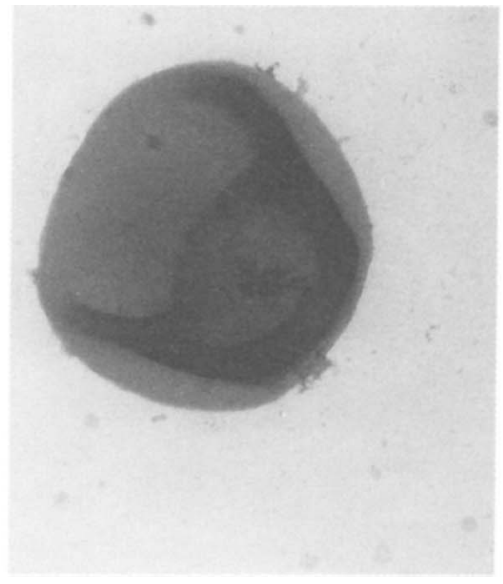

4223NC

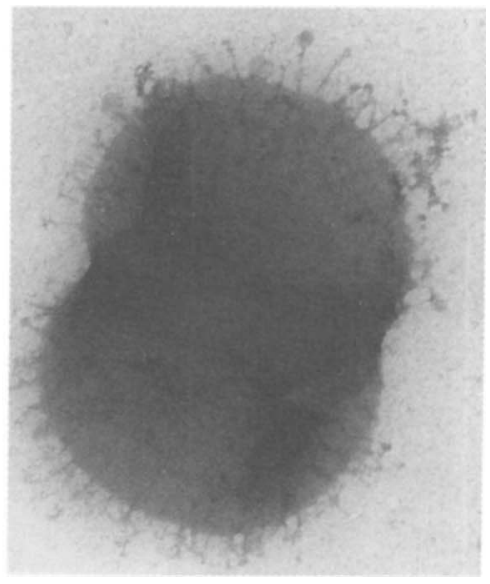

K114

Fig. 5. Electron micrographs of $M$. catarrhalis strains 4223, 4223NC and K114 as noted. Magnification, $\times 34000$. 

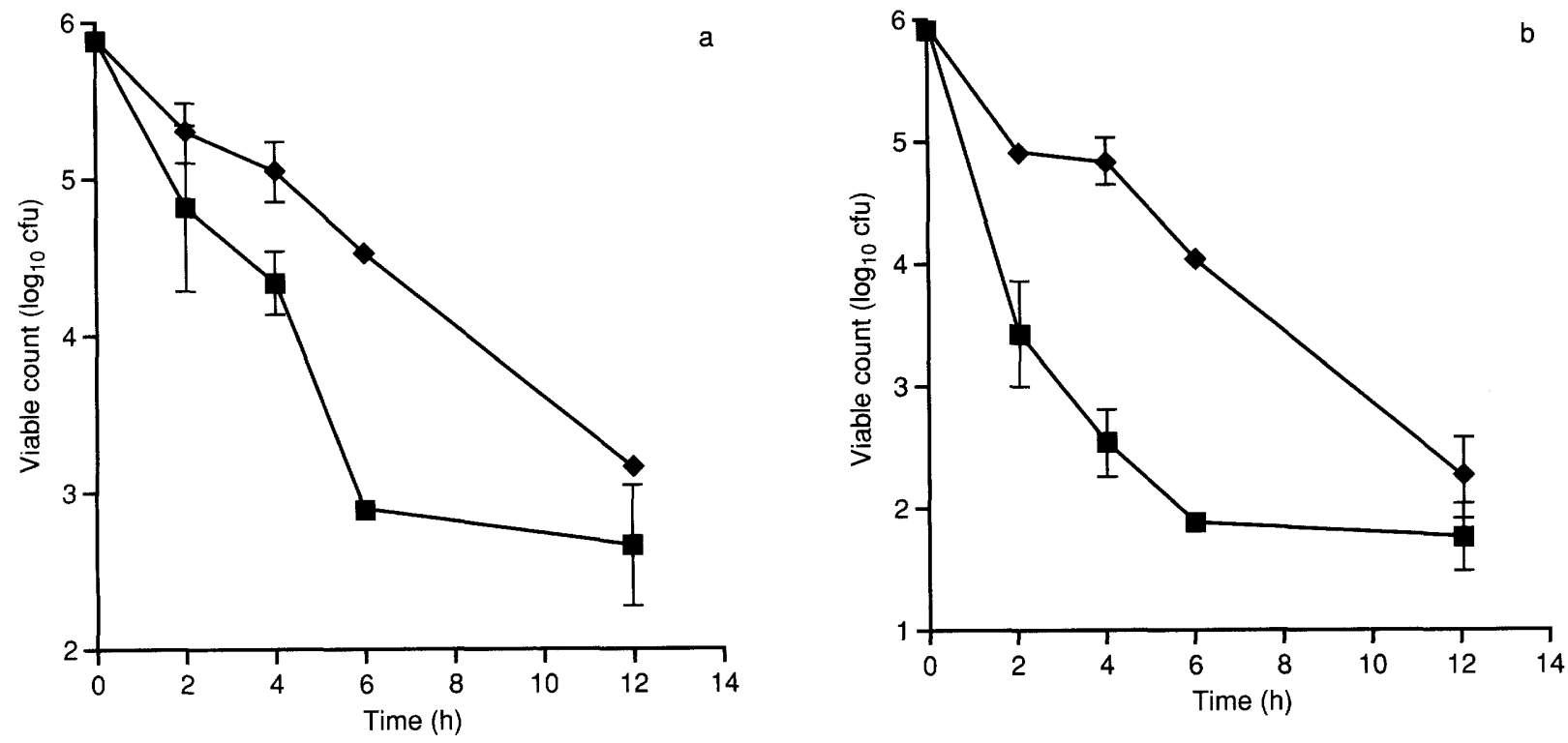

Fig. 6. Bacterial recovery from $\mathbf{a}$, lung and $\mathbf{b}$, bronchoalveolar lavage fluid (BAL) following intratracheal challenge of mice with $10^{6} \mathrm{cfu}$ of $M$. catarrhalis strains $4223(\mathbf{\square})$ and $4223 \mathrm{NC}(\bullet)$ as noted. The y-axes indicate the $\log _{10} \mathrm{cfu}$ from lung or BAL. The $\mathrm{x}$-axes indicate time after challenge in $\mathrm{h}$. Each point represents the mean and SEM for samples from four or five animals. For points without error bars, the SEM is too small to be depicted.
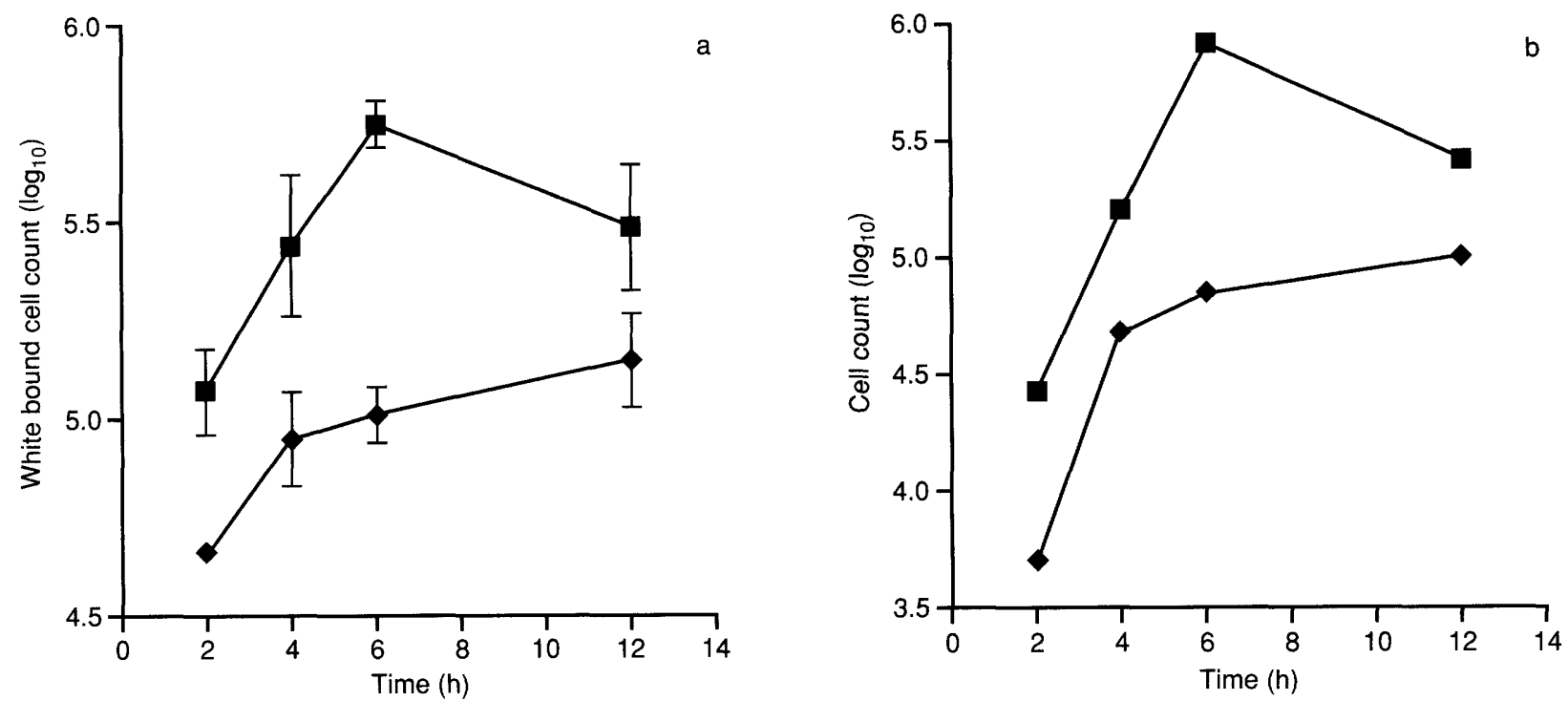

Fig. 7. a, White blood cell counts and b, neutrophil counts in bronchoalveolar lavage fluid (BAL) following intratracheal challenge of mice with $10^{6} \mathrm{cfu}$ of $M$. catarrhalis strains $4223(\mathbf{E})$ and $4223 \mathrm{NC}(\diamond)$ as noted. The y-axes indicate the $\log _{10}$ cell count in BAL. The $\mathrm{x}$-axes indicate time after challenge in $\mathrm{h}$. Each point represents the mean and SEM for samples from four or five animals.

appeared to express similar amounts of OMP CD in their outer membranes; (4) alteration of surface accessibility of an epitope on LOS of the nonclumping variant in spite of similar LOS banding patterns of both strains.

An obvious difference between the two strains was the markedly reduced expression of the HMW-OMP in the non-clumping variant strain. The reactivity of the band with MAb 9E9 in immunoblot assay and flow cytometry confirmed the identity of the band as the
HMW-OMP which is also called UspA $[7,9,15]$. The function of this protein is not yet known, although preliminary data suggest that it may function as an adhesin.

The identity of the protein band of c. $200 \mathrm{kDa}$ which is absent from the non-clumping variant strain is not known. Molecular mass estimates in this size range are inaccurate. The possibility that this band represented the $120-\mathrm{kDa}$ form of the HMW-OMP which has been observed $[9,15]$ was considered. This 
possibility seems unlikely for two reasons. First, the band appears to be substantially higher than $120 \mathrm{kDa}$ in molecular mass. Second, the band is not reactive with MAb 9E9 which recognises the $120-\mathrm{kDa}$ form of the HMW-OMP [15]. It is more likely that the c. 200$\mathrm{kDa}$ band represents a protein which is distinct from the HMW-OMP.

An interesting result was observed with flow cytometry with MAbs that recognise different epitopes on OMP CD. MAb $3.9 \mathrm{H}$, which binds a surface epitope near the amino terminus of the $\mathrm{CD}$ molecule, yielded identical results with the two strains. By contrast, $\mathrm{MAb} 1 \mathrm{D} 3$, which binds a surface epitope in the central region of the $C D$ molecule, showed reduced binding to the non-clumping variant compared to the parent strain (Fig. 3). As the two strains appear to express the same amount of OMP CD, it seems likely that the outer-membrane architecture of strain $4223 \mathrm{NC}$ has been altered in such a way as to change the surface accessibility of the central region of OMP CD.

A similar observation regarding the LOS of the two strains was made. The banding patterns in SDS-PAGE of LOS purified from the two strains were identical. However, flow cytometry with a MAb that binds an epitope on the bacterial surface revealed alteration in the surface accessibility of that epitope.

Outer-membrane antigens were altered selectively by in-vitro passage of $M$. catarrhalis in broth from which aggregates had settled because many surface antigens were apparently unaffected. Electron microscopic examination of the strains 4223 and $4223 \mathrm{NC}$ revealed an absence of pili in both strains and no apparent morphological differences. Analysis of the two strains by flow cytometry with MAbs that recognise epitopes on two other major OMPs (OMPs B2 and E) showed no differences in the availability of these epitopes on the bacterial surface.

Differences in bacterial clearance between the variant strains were observed following pulmonary challenge of mice. This difference was particularly prominent in BAL samples, from which the non-clumping variant was consistently recovered in higher numbers than the parent strain. The ratio of bacteria in BAL to lung was higher for the non-clumping variant at all time points. The bacteria which were recovered from BAL represent those which remained in the airways; they did not invade into the lung and did not adhere to respiratory epithelium tightly enough to resist removal by a single bronchoalveolar lavage. To explain the observation of increased numbers of the non-clumping variant in BAL samples, it could be speculated that the non-clumping strain shows reduced adherence to respiratory epithelium. Less adherence may lead to less invasion into the lung tissue. Therefore, greater numbers of the non-clumping strain are recovered from the BAL. A further possibility is that clumps of the parent strain are more readily phagocytosed than single cells of the non-clumping strain. Therefore, the number of wild-type organisms is reduced more quickly. It is not yet possible to conclude whether these mechanisms or others account for the reduced pulmonary clearance of the non-clumping strain.

The rate of recruitment of neutrophils to the lung is important for the early clearance of non-typable Haemophilus influenzae and Pseudomonas aeruginosa $[20,21]$. The present study also shows a correlation between recruitment of neutrophils to the lung and pulmonary clearance of $M$. catarrhalis. The identification and significance of factors contributing to the reduced numbers of neutrophils recruited to the lungs in response to challenge with the non-clumping variant have yet to be determined.

The present study shows that alteration of expression of surface molecules has a significant impact in an invivo model of pulmonary clearance of $M$. catarrhalis. Caution must be observed in drawing conclusions regarding which molecule(s) is (are) responsible for this observation. Clearly, in-vitro passage of $M$. catarrhalis results in important effects on the outer membrane and the behaviour of the bacterium in the pulmonary clearance model. These results emphasise the importance of remaining aware of the effects of in-vitro passage of $M$. catarrhalis as this model is used to test potential vaccine antigens. Future work will focus on identifying the functions of the bacterial molecules responsible for these differences, the mechanisms by which $M$. catarrhalis alters expression of surface molecules, and the host mechanisms by which $M$. catarrhalis is cleared from the respiratory tract. The identification of a bacterial molecule that is capable of modulating the host immune response may provide insight into changes that could occur during transition from a commensal organism to one that causes infection.

This work was supported by funds from the University of Canberra visiting fellowship scheme and by grant AI28304 from the National Institutes of Health. T.F.M. was supported by Senior International Fellowship TW02158 from the Fogarty International Center of the National Institutes of Health. We thank Ruth Foxwell for assistance with bacteriology, and Charmaine Kirkham, Norine Yuskiw and David Smail for expert technical assistance.

\section{References}

1. Murphy TF. Branhamella catarrhalis: epidemiology, surface antigenic structure, and immune response. Microbiol Rev 1996 60: $267-279$.

2. Murphy TF, Sethi S. Bacterial infection in chronic obstructive pulmonary disease. Am Rev Respir Dis 1992; 146: 1067-1083.

3. Verghese A, Roberson D, Kalbfleisch JH, Sarubbi F. Randomized comparative study of cefixime versus cephalexin in acute bacterial exacerbations of chronic bronchitis. Antimicrob Agents Chemother 1990; 34: 1041-1044.

4. Unhanand M, Maciver I, Ramilo $\mathrm{O}$ et al. Pulmonary clearance of Moraxella catarrhalis in an animal model. $J$ Infect Dis 1992; 165: 644-650. 
5. Verghese A, Berro E, Berro J, Franzus BW. Pulmonary clearance and phagocytic cell response in a murine model of Branhamella catarrhalis infection. $J$ Infect Dis 1990; 162: 1189-1192

6. Maciver I, Unhanand M, McCracken GH, Hansen EJ. Effect of immunization on pulmonary clearance of Moraxella catarrhalis in an animal model. $J$ Infect Dis 1993; 168: 469-472.

7. Helminen ME, Maciver I, Latimer JL et al. A large, antigenically conserved protein on the surface of Moraxella catarrhalis is a target for protective antibodies. $J$ Infect Dis 1994; 170: 867-872.

8. Helminen ME, Maciver I, Latimer JL, Cope LD, McCracken $\mathrm{GH}$, Hansen EJ. A major outer membrane protein of Moraxella catarrhalis is a target for antibodies that enhance pulmonary clearance of the pathogen in an animal model. Infect Immun 1993; 61: 2003-2010.

9. Chen D, McMichael JC, VanDerMeid KR et al. Evaluation of purified UspA from Moraxella catarrhalis as a vaccine in a murine model after active immunization. Infect Immun 1996; 64: $1900-1905$.

10. Murphy TF, Bartos LC. Surface-exposed and antigenically conserved determinants of outer membrane proteins of Branhamella catarrhalis. Infect Immun 1989; 57: 2938-2941.

11. Inzana TJ. Electrophoretic heterogeneity and interstrain variation of the lipopolysaccharide of Haemophilus influenzae. $J$ Infect Dis 1983; 148: 492-499.

12. Murphy TF, Dudas KC, Mylotte JM, Apicella MA. A subtyping system for nontypable Haemophilus influenzae based on outer-membrane proteins. $J$ Infect Dis 1983; 147: 838-846.

13. Lesse AJ, Campagnari AA, Bittner WE, Apicella MA. Increased resolution of lipopolysaccharides and lipooligosaccharides utilizing tricine-sodium dodecyl sulfate-polyacryla- mide gel electrophoresis. J Immunol Methods 1990; 126: 109-117.

14. Murphy TF, Bartos LC, Campagnari AA, Nelson MB, Apicella MA. Antigenic characterization of the P6 protein of nontypable Haemophilus influenzae. Infect Immun 1986; 54: 774-779.

15. Klingman KL, Murphy TF. Purification and characterization of a high-molecular-weight outer membrane protein of Moraxella (Branhamella) catarrhalis. Infect Immun 1994; 62: 1150-1115.

16. Sarwar J, Campagnari AA, Kirkham C, Murphy TF. Characterization of an antigenically conserved heat-modifiable major outer membrane protein of Branhamella catarrhalis. Infect Immun 1992; 60: 804-809.

17. Klingman KL, Pye A, Murphy TF, Hill SL. Dynamics of respiratory tract colonization by Branhamella catarrhalis in bronchiectasis. Am J Respir Crit Care Med 1995; 152: 10721078 .

18. Sethi S, Surface JM, Murphy TF. Antigenic heterogeneity and molecular analysis of CopB of Branhamella (Moraxella) catarrhalis. Infect Immun 1997 (in press)

19. Bhushan R, Kirkham C, Sethi S, Murphy TF. Antigenic characterization and analysis of the human immune response to outer membrane protein E of Branhamella catarrhalis. Infect Immun 1997; 65: 2668-2675.

20. Buret A, Dunkley M, Clancy RL, Cripps AW. Effector mechanisms of intestinally induced immunity to Pseudomonas aeruginosa in the rat lung: role of neutrophils and leukotriene B $_{4}$. Infect Immun 1993; 61: 671-679.

21. Kyd JM, Dunkley ML, Cripps AW. Enhanced respiratory clearance of nontypeable Haemophilus influenzae following mucosal immunization with P6 in a rat model. Infect Immun 1995; 63: $2931-2940$. 